\title{
Heterogeneidad territorial de las políticas públicas DE PROTECCIÓN SOCIAL: EL CASO DE LAS RENTAS MÍNIMAS DE INSERCIÓN EN ESPAÑA
}

\author{
Territorial Heterogeneity of Public Social Protection Policies: The Case \\ of Minimum Insertion Income in Spain
}

\author{
CARMEN RODRÍGUEZ SUMAZA
}

Universidad de Valladolid, España

\section{MARÍA DE LAS MERCEDES PRIETO ALAIZ}

Universidad de Valladolid, España

\section{JUAN MARÍA PRIETO LOBATO}

Universidad de Valladolid, España

\author{
JESÚS GARCÍA-ARAQUE
}

Universidad de Valladolid, España

\begin{abstract}
RESUMEN
Las comunidades autónomas españolas han ido desarrollando en las últimas décadas diferentes programas regionales de garantía de rentas que se enmarcan en el contexto de las políticas públicas para combatir la pobreza y la exclusión social y que incluyen unas rentas mínimas de inserción. El desarrollo territorial de este último recurso ha resultado desigual, conformándose así sistemas regionales con diferente grado de generosidad, cobertura, características y alcance. En este trabajo se analizan empíricamente esas diferencias a partir de la aplicación de la técnica de análisis de conglomerados. Los resultados confirman, junto a la relevancia del recurso, la necesidad de reorientar el sistema en aras de la consecución de un modelo territorial equilibrado y garante de la igualdad de derechos y oportunidades de todas las personas.
\end{abstract}

Palabras clave: Políticas públicas, Rentas Mínimas de Inserción, España.

\begin{abstract}
Spanish regional governments have developed in the last decades different income-guarantee programs within the public policies to tackle poverty and social exclusion. They all include a minimum insertion income scheme. The territorial development of these schemes has been unequal, thus leading to regional systems with different degrees of generosity, coverage, characteristics, and scope. In this work, we analyze empirically these differences with a cluster analysis technique. The results confirm, together with the relevance of the resource, the need to reshape the system in order to achieve a more balanced territorial model able to ensure equal rights and opportunities for all people.
\end{abstract}

Keywords: Public Policies, Minimum Insertion Income, Spain. 


\section{INTRODUCCIÓN. LAS RENTAS MÍNIMAS DE INSERCION EN EL CONTEXTO DEL SISTEMA DE GARANTÍA DE INGRESOS}

El sistema de protección social del Estado español está constituido por un complejo entramado de recursos orientados a garantizar a las personas el ejercicio de sus derechos y deberes constitucionales. Este sistema consta de dos niveles, uno de carácter contributivo -en el que la protección de los sujetos está vinculada a sus aportaciones previas-, y otro de carácter asistencial o no contributivo -orientado a asegurar unos ingresos mínimos tanto a las personas trabajadoras que ya han agotado la protección contributiva como a las personas que no pueden trabajar por razón de edad, enfermedad o discapacidad-. En este segundo nivel, de carácter complementario, están integradas toda una serie de prestaciones especialmente orientadas a garantizar unos ingresos mínimos, fundamentalmente por la vía de promover la reducción de la pobreza y la activación laboral.

En el desarrollo de este sistema español de garantía de rentas están implicados dos ámbitos administrativos. Por un lado, el Estado español es el encargado de regular "las condiciones básicas que garanticen la igualdad de todos los españoles" (Constitución Española, 1978: art. 149.1); por otro lado, se encuentran las 17 comunidades autónomas (CCAA, en adelante) que conforman el Estado español, y que han ido progresivamente asumiendo competencias en materia de servicios sociales al hilo del desarrollo de sus respectivos Estatutos de Autonomía. El sistema, que comprende en la actualidad una variada gama de prestaciones y complementos que financia y gestiona en su mayoría el Estado a través de diferentes instituciones, incluye las denominadas rentas mínimas de inserción (RMI, en adelante). Estas RMI, objeto específico de estudio de este trabajo, son prestaciones económicas periódicas de ámbito autonómico orientadas a paliar la pobreza por la vía de la promoción de la integración laboral y social de los individuos, es decir, buscan garantizar la cobertura de sus necesidades básicas durante el proceso de inserción. Junto a su carácter asistencial, se caracterizan también por ser subsidiarias, complementarias y no universales (son un último recurso para aquellos con prestaciones insuficientes o que no acceden a otras prestaciones sociales, y su cuantía varía en función de la situación económica del demandante y el tope máximo establecido), vinculándose su percepción a la participación en un proceso personalizado de inserción laboral.

Las RMI se implantaron progresivamente en España en los años noventa tomando como referencia principal el modelo de Revenu Minimum d'Insertion vigente en Francia desde 1988, que combinaba una prestación económica de cuantía diferencial y carácter complementario destinada a personas sin recursos y sin derecho al desempleo con un proyecto de inserción laboral. Aquí, sin embargo, el Estado era el encargado de financiar la prestación y los consejos departamentales los responsables de las acciones de integración. Este modelo inicial dio paso en 2009 a la actual Revenu de Solidarité Active, un recurso más descentralizado, al ser gestionado por los consejos departamentales, y que re- 
fuerza los compromisos de inserción de los beneficiarios a la vez que contempla mayores incentivos para el empleo, pues permite compatibilizar la prestación con las rentas del trabajo si éstas son bajas, mejora los servicios personalizados de acompañamiento para el empleo y complementa éstos con ayudas para compatibilizar la vida laboral y familiar.

En su conjunto, el entramado existente de recursos del sistema español de garantía de ingresos y su compleja organización dan lugar a un sistema con "problemas de articulación" entre prestaciones y administraciones implicadas (Ayala et al 2016: 9) que algunos informes no dudan en calificar como "fracturado e incoherente" (Malgesini 2014: 7) y "poco sistemático." Existe, de hecho, un cierto consenso en torno a la idea de que, a pesar de que todas las prestaciones comparten un mismo objetivo, "constituyen más bien un mosaico de figuras que un 'sistema' (Galiano et al 2014: 59) en el que los recursos se limitan a convivir entre sí generando "redes paralelas de intervención, más o menos complementarias, con muy escasos elementos de coordinación e integración funcional entre sí" (Sanzo 2018a: 42). Como veremos más adelante, esta configuración tiene consecuencias importantes en términos de impacto y eficiencia en la reducción de la pobreza y la desigualdad (Laparra y Ayala 2009: 19).

A los problemas anteriormente mencionados se añade el hecho de que las RMI, al depender exclusivamente de las administraciones regionales, presentan rasgos diferenciadores en términos de cuantías, criterios, requisitos, duración, etc., lo que genera una importante diversidad territorial y añade una debilidad adicional al sistema. El análisis de esta última cuestión constituye el objetivo principal de este trabajo, que se justifica principalmente, por un lado, por el interés que tiene el conocimiento en el panorama internacional de los elementos que caracterizan los sistemas singulares de garantía de rentas en contextos como el actual de progresiva descentralización del Estado del Bienestar y de creciente apuesta por la activación de las políticas de inserción y, por otro, por la necesidad de disponer de conocimiento empírico preciso sobre la eficacia de estos sistemas que pueda permitir reformular y mejorar la articulación de las estrategias de lucha contra la exclusión, particularmente en situaciones de creciente demanda de ayuda social. Cabe señalar también, en relación a esta última cuestión, la oportunidad de contribuir al debate sobre el papel que pueden seguir desempeñando las rentas mínimas autonómicas en el contexto español actual de inminente puesta en marcha de un Ingreso Mínimo Vital a cargo del Estado (Sanzo 2020) o, con carácter más general, sobre la pertinencia o no de transitar a un modelo de renta básica universal en el que todos los individuos, sin condiciones, tienen derecho a percibir una cantidad orientada a cubrir sus necesidades básicas en condiciones que garantizan su libertad para desarrollarse como persona.

El trabajo parte de una revisión en profundidad de los principales estudios existentes con el fin de desentrañar los asuntos que han centrado el interés de investigadores y personas expertas que han explorado en los últimos años la diversidad territorial derivada de la coexistencia de sistemas autonómicos de 
garantía de rentas muy diferentes en cuanto a nivel de desarrollo, alcance y cobertura. En segundo lugar, y una vez constatada la ausencia en el contexto español de un trabajo de carácter empírico que aborde con especial rigor estadístico la compleja y diversa panorámica regional, se procede al empleo de la técnica de análisis clúster para establecer agrupaciones de CCAA con rasgos homogéneos en lo que a sus RMI se refiere y para caracterizar las diferencias entre unos y otros grupos. Tras exponer los resultados obtenidos confirmando la existencia de sistemas diversos de garantías de ingresos mínimos que configuran un preocupante panorama desigual en la actualidad, se presentan las conclusiones del trabajo, que apuntan, entre otras cuestiones, a la necesidad de emprender reformas significativas tendentes a la racionalización del sistema.

\section{EL ESTUDIO DE LAS DIFERENCIAS TERRITORIALES DE LAS RMI. ESTADO DE LA CUESTIÓN}

Las diferencias territoriales en materia de RMI en España vienen siendo objeto recurrente de estudio desde hace años por parte de investigadores y estudiosos de la política social, siendo Aguilar, Gaviria y Laparra (1994) uno de los trabajos pioneros. Una revisión en profundidad de los estudios más relevantes realizados en los últimos años permite identificar las siguientes grandes cuestiones en torno a las cuales giran dichos análisis.

Una de las cuestiones que ha suscitado mayor interés es el esfuerzo presupuestario realizado por las CCAA, es decir, los recursos económicos que en cada lugar y momento se han ido destinado a las RMI. Esta cuestión se ha abordado fundamentalmente de dos maneras: (a) analizando el nivel y la evolución del gasto anual en RMI, tanto en términos absolutos como relativos (con respecto al total de titulares, de beneficiarios o de habitantes); y (b) estudiando el esfuerzo presupuestario de cada CA por la vía de comparar dicho gasto con el PIB y/o el total del presupuesto regional. Los resultados son lógicamente diversos según los períodos de tiempo analizados. Como señala Arriba (2014), el sistema ha estado sometido durante la crisis a una gran tensión derivada, por un lado, de una creciente demanda de ayudas y, por otro, de la puesta en marcha de políticas de consolidación fiscal y austeridad limitadoras del gasto. No obstante, los dispersos itinerarios del esfuerzo presupuestario de las CCAA han de explicarse también teniendo en consideración los "cambios institucionales (en la regulación) o decisiones puntuales de los ejecutivos autonómicos" (Arriba, 2014: 11). Por lo general, durante el periodo de crisis económica las diferentes CCAA han incrementado el volumen de recursos destinados a las RMI. Así lo constata, entre otros, Herranz (2017) al observar que entre 2007 y 2015 se triplicó el volumen de gasto, dado que las RMI llegaron a más gente y con cuantías más elevadas. Para Fernández (2013: 172), sin embargo, resulta necesario corregir las cifras oficiales de incremento del gasto en RMI con el IPC acumulado, lo que permite comprobar que en periodos como el 2008-2011 no se puede afirmar realmente que estas prestaciones hayan estado "resistiendo la 
embestida de la crisis." Asimismo, parece comprobarse que, salvo en el caso del País Vasco, el esfuerzo presupuestario de las diferentes CCAA es generalmente bastante reducido (Arriba y Pérez 2007) y "el compromiso presupuestario no es siempre proporcional a las necesidades y a la población de cada comunidad" (Noguera 2019: 7).

Junto al tema estrictamente económico, un segundo asunto de análisis preferente en relación con las RMI ha sido el estudio de las variaciones en sus características básicas (condiciones de acceso, duración de las prestaciones, etc.). En el período inicial de la crisis (2008-2011), las CCAA procedieron en general a un cierto endurecimiento de los requisitos de acceso a las RMI, especialmente en el caso de la prueba de rentas y la duración de la prestación, quizás para compensar los limitados recursos disponibles. Esta circunstancia obliga a hablar de un debilitamiento o 'aumento de la fragilidad' de las RMI durante dicho período (Fernández, 2013). Por su parte, Bergantiños et al (2017), fijándose expresamente en la cuantía, duración, garantías de financiación (si es derecho subjetivo o no) y las condiciones de acceso a la prestación, observan una gran heterogeneidad de situaciones, resultando más numerosos los casos de CCAA que empeoran durante el período 2008-2014, especialmente las que tenían inicialmente unas RMI más débiles. Estudios comparativos más recientes, como los realizados por Noguera (2018), Moreno (2018), Aguilar y Arriba (2019) y Valdueza (2019), confirman que gobiernos regionales de diferente color político han venido desplegando en el tiempo unas nuevas rentas mínimas con requisitos más relajados (prácticamente en todas las reformas emprendidas desde 2015), más generosas y con interesantes avances en su diseño y gestión (especialmente en las CCAA del País Vasco, Navarra, Valencia y Cataluña). Entre las novedades principales de esta nueva generación de rentas mínimas están la "incondicionalidad de la prestación respecto de la firma de un plan de inserción" y la posibilidad de que se compatibilicen las prestaciones con unos ciertos ingresos salariales (Noguera 2019: 14).

Un tercer asunto sometido a análisis en buena parte de los estudios sobre RMI es el relativo a su nivel de cobertura, reflejo del interés por analizar a cuántas personas llegan las prestaciones y si éstas son ciertamente las personas que más lo necesitan. El análisis practicado por Rodríguez (2009) previo a la crisis económica ya identificaba, en aras de la mejora del sistema y, entre otras cuestiones, la necesidad de conseguir un acercamiento progresivo de las prestaciones a los umbrales de pobreza relativa. Si bien la crisis económica ha supuesto en todos los territorios un incremento muy significativo tanto del número de titulares como de los beneficiarios indirectos, Fernández (2013: 169) comprueba, para el período 2008-2011, que, a pesar de ello, el sistema "solo llega a un tercio de los hogares que lo necesitarían." Por su parte, Malgesini (2014) constata una falta de cobertura de las rentas mínimas en el caso de ciertos colectivos vulnerables (inmigrantes irregulares, personas sin hogar, ocupas, personas mayores de 65 años, personas con discapacidad, etc.). Otros autores, como Herranz (2017), llaman la atención acerca de que el perfil de los beneficiarios, tradicionalmente 
vinculados de forma mayoritaria a situaciones de exclusión, se ha visto alterado con la crisis económica al incorporarse también personas en situación de pobreza monetaria. Asimismo, hacen notar que las cifras de titulares de la prestación no son proporcionales al tamaño de la población de los territorios y las tasas de cobertura de la prestación ${ }^{1}$ son extraordinariamente diferentes entre regiones. Todo ello estaría reflejando la diferente "apuesta que cada uno de los gobiernos autonómicos ha decidido realizar por la implementación de este ámbito de protección social" (Herranz, 2017: 89) y explica datos tan significativos como que para 2015 se concentren uno de cada cuatro beneficiarios de RMI en el País Vasco, o que esta CA y Navarra presenten tasas de cobertura muy por encima de la media regional.

En esta misma línea de análisis, Bergantiños et al. (2017), interesados en analizar las tasas de cobertura sobre la población en riesgo de pobreza, ${ }^{2}$ constatan una mejora en la mayoría de las CCAA entre 2008 y 2014, si bien es cierto que se partía de valores muy diferentes y en algunos casos realmente bajos. Por su parte, Aguilar y Arriba (2019) destacan que la cobertura, tanto sobre la población en su conjunto como sobre la población en una situación de pobreza relativa de cada territorio, es muy superior en tres CCAA (País Vasco, Navarra y Asturias), si bien el crecimiento relativo en el resto ha sido importante (si en 2008 esas tres CCAA representaban un 43.3\% del total de RMI, en 2017 su peso había caído al 36.5\%). En cualquier caso, y salvo las citadas excepciones, se confirma una cobertura aun desigual y "todavía insuficiente de las necesidades de los colectivos" (Aguilar y Arriba, 2019: 414). Esta circunstancia que, según Laparra $(2013 ; 2020)$, se explica no tanto por las condiciones monetarias de estos programas (aunque sean generosas) como por su propia concepción y por los modelos de gestión adoptados (que incluyen importantes frenos y limitaciones de acceso y mantenimiento), es una de las razones de la ineficiencia del modelo español en la reducción de la pobreza y, especialmente, de la pobreza severa.

Otro relevante asunto que ha centrado la atención de los estudiosos de las RMI es el tema de la cuantía de las prestaciones, es decir, el asunto de su intensidad protectora. Esta cuestión puede ser valorada de muy diferentes maneras, si bien en la mayoría de los trabajos se relaciona la cuantía de las RMI (incluyendo la cuantía básica y sus complementos en función de los miembros del hogar) con otras referencias económicas: el salario mínimo interprofesional, IPREM, la cuantía de las pensiones no contributivas, el umbral de pobreza relativa y/o la renta familiar. En este sentido, hace ya unos años Rodríguez (2009:16) concluía que la extensión de los sistemas de RMI no había estado acompañada de una "adecuada intensidad protectora medida en relación al salario mínimo, el umbral de renta y la renta familiar per cápita disponible" y, en consecuencia, que estos sistemas habían demostrado una limitada contribución a la reducción

Medidas éstas como la relación entre los titulares y los beneficiarios y la población total.

Que relacionan las cifras de beneficiarios con el número de personas en riesgo de pobreza (extraídas de la Encuesta de Condiciones de Vida) en cada región. 
de las tasas de pobreza relativa y a la aproximación a los niveles de renta per cápita disponible. Análisis posteriores, como los de Arriba (2009; 2014), han venido a confirmar una desigual intensidad protectora en las diferentes regiones: con CCAA como Murcia y Ceuta garantizando cuantías bien por debajo de la media nacional, mientras que otras, como Cataluña, Navarra y el País Vasco, resultaban mucho más generosas. Por último, en relación a la suficiencia de las cuantías de las prestaciones, el informe del CES observa en general la ausencia "de una referencia básica que actúe como una especie de 'nivel de dignidad' (...) suficiente para cubrir las necesidades de un individuo o familia" (CES 2017: 87).

El quinto y último objeto de análisis en los trabajos que analizan las RMI es su efectividad, considerada en términos de la capacidad o impacto que estas rentas tienen a la hora de conseguir dos de sus objetivos fundamentales: la reducción de la pobreza y el fomento de la inserción laboral de sus perceptores. Respecto al primer objetivo, Rodríguez (2009: 18), califica de "limitada" la capacidad del sistema español para reducir la pobreza, lo que exigiría no solo su mejora sino su compatibilidad con otras prestaciones e ingresos. En esa misma línea, Noguera (2019: 16) ha advertido que "no puede decirse que su impacto en la reducción de la pobreza o la desigualdad en general sea muy voluminoso en comparación con el que tienen otras prestaciones monetarias como las pensiones o las prestaciones por desempleo." Respecto al segundo objetivo, aunque solo se cuenta con las evaluaciones de algunos programas autonómicos específicos (Madrid, Cataluña y País Vasco, entre otros), algunos acercamientos empíricos permiten afirmar con cierta seguridad que las rentas mínimas tienen un bajo éxito en cuanto al acceso al mercado de trabajo, pero que no hay suficiente evidencia que fundamente la idea de que desincentivan la búsqueda de empleo y de que generan problemas de cronificación (Ayala 2016: 156). En este sentido, Riba, Ballart y Blasco (2011) han confirmado que no solo estas prestaciones no retrasan la entrada en el empleo, sino que la aceleran en el caso de personas con educación media y alta y para los mayores de 45 años. Asimismo, otros trabajos han evidenciado que la participación en itinerarios de inserción laboral aumenta la probabilidad de encontrar empleo, si bien la existencia de diferentes perfiles sociolaborales exige diferentes modelos de intervención, incrementos de la intensidad y un enfoque a largo plazo (Casado 2010). Recientemente, la AIReF (2019), a propuesta del Parlamento de España, ha llevado a cabo un minucioso análisis del sistema de rentas mínimas que evidencia "debilidades en su diseño que repercuten en la eficacia, institucionalidad y eficiencia" (AIReF, 2019: 5).

En general, los análisis de los efectos del sistema de garantía de ingresos sobre el nivel de pobreza vienen a demostrar que, aunque en su conjunto el sistema "ha mostrado una notable capacidad de ajuste al cambio de ciclo económico, no ha resultado suficiente para dar respuesta al veloz aumento de las situaciones de insuficiencia de ingresos en la crisis" (Ayala et al 2016: 13). En este contexto, el importante crecimiento experimentado por las rentas mínimas autonómicas ha 
permitido, en cierto modo, compensar las carencias de un limitado y 'fracasado' sistema de nacional de protección (Sanzo 2018a). Esa compensación resulta ser, sin embargo, limitada y desigual. Limitada por lo exiguo de los recursos propios de las RMI y desigual por el muy diferente alcance que éstos tienen en los diferentes territorios. En relación con el primero de los asuntos, y a pesar de la polémica que, en el plano político y social, genera en ocasiones este recurso, el Gráfico 1 permite ver que los perceptores titulares de rentas mínimas de inserción supusieron en 2017 poco más de un 5\% del total de perceptores del sistema de garantía de ingresos mínimos. De forma similar, el gasto en rentas mínimas de inserción supuso tan solo un $7.6 \%$ del total de euros que invirtió el sistema en su conjunto en dicho año. La segunda de las cuestiones mencionadas es objeto específico de análisis en los siguientes apartados.

Gráfico 1. Porcentaje de gasto anual y de número de perceptores de las prestaciones del sistema de garantía de ingresos mínimos, 2017.

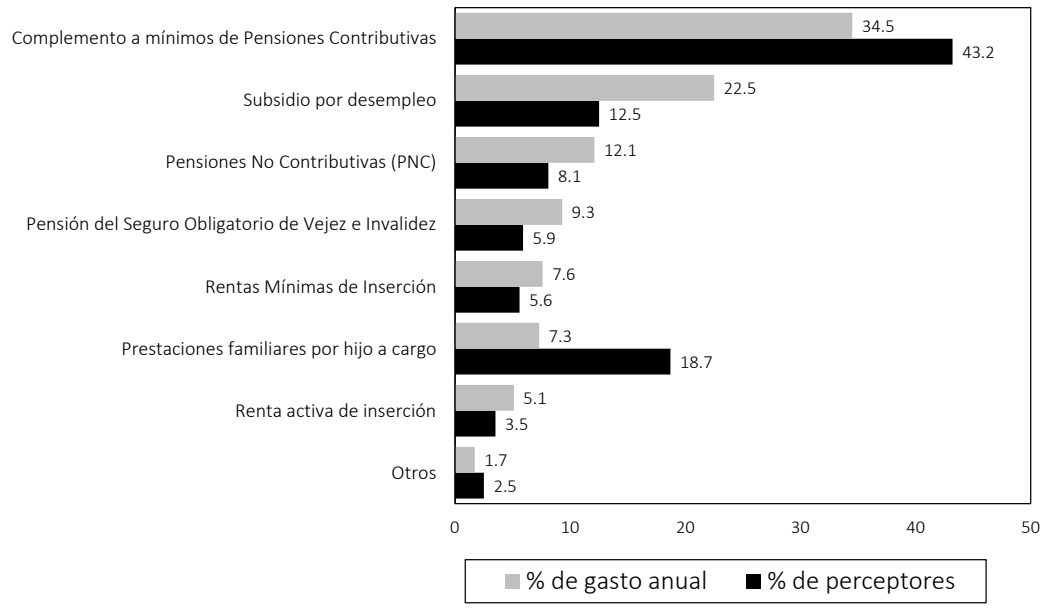

Fuente: Elaboración propia con datos de MSCBS (2019).

Por lo que respecta al contenido, los principales estudios sobre las RMI en las diferentes CCAA que se han repasado coinciden en caracterizar unos sistemas asistenciales regionales muy diversos que conforman una panorámica territorial históricamente muy desigual en la que sistemáticamente destacan los casos del País Vasco y Navarra, comunidades que presentan los más altos niveles de desarrollo del sistema. ${ }^{3}$ Los trabajos analizados permiten asimismo evidenciar los aspectos en los que se manifiestan en mayor grado las desigualdades regionales, apuntando con ello las dimensiones a atender y a considerar de una 
manera más decidida de cara a la mejora del conjunto del sistema. ${ }^{4}$ En términos generales, el repaso de la literatura científica pone en evidencia que desde hace tiempo se reclama una revisión en profundidad de la política española de garantía de rentas (Laparra y Ayala 2009; Montes 2020), el desarrollo de normas reguladoras del papel de las CCAA (Barragué y Martínez 2012) e, incluso, la implantación alternativa de una renta básica (Sanzo 2018b).

Desde una perspectiva metodológica, todos los estudios analizados toman como base de partida la información oficial que periódicamente proporcionan los informes de RMI (MSCBS, varios años), predominando los trabajos de carácter descriptivo que comparan en fechas concretas los datos por CCAA y/o que estudian la variación experimentada en el tiempo por los mismos. Estos trabajos permiten fundamentalmente ordenar a las distintas CCAA en base a los diferentes atributos de su sistema de RMI e identificar aquellas que en cada momento están mejor y/o peor posicionadas. Algunos trabajos van un poco más allá e intentan identificar grupos de CCAA en cuanto a las características y el alcance de sus sistemas de RMI (Aguilar et al 1994; Martínez 2005; Arriba 2014; Herranz 2017; Bergantiños et al 2017). Se echa en falta, sin embargo, un análisis que, apoyado más sólidamente en técnicas estadísticas, permita identificar y fundamentar, si es el caso, la existencia de grupos relativamente homogéneos de CCAA en lo que a sus sistemas de RMI se refiere. Esto es lo que se pretende hacer a continuación, en un intento por contribuir a mejorar el conocimiento de las características y de los rasgos fundamentales del sistema español de RMI.

\section{DATOS Y METODOLOGÍA}

\section{Indicadores y fuentes de información.}

Como se ha reflejado en el apartado anterior, en los últimos años se han realizado en España muy diversos trabajos que, centrados en algunos aspectos concretos de las RMI, buscan caracterizar los cambios más significativos experimentados por este recurso. Todos ellos concluyen que la variabilidad intra e intersistemas autonómicos de RMI en los diferentes períodos de su desarrollo ha sido enorme; algunos apuntan, además, la idea de que tal diversidad se debe a la heterogeneidad en la respuesta política al problema de la pobreza (AIRef 2019). 
El objetivo de este trabajo, tal y como se ha señalado con anterioridad, no es realizar un examen evolutivo de estos sistemas, ${ }^{5}$ sino analizar su configuración en el momento actual (los últimos datos disponibles corresponden al año 2017), identificar sus elementos comunes y diferenciales y, según ellos, acometer un ejercicio taxonómico que mejore su conocimiento y comprensión. Con este fin, se ha optado por utilizar una batería de 10 variables de naturaleza tanto absoluta como relativa, cuya descripción se muestra en la Tabla 1 y cuyos valores pueden consultarse en el Anexo I. En los indicadores de tipo relativo, se ha tomado como referencia el comportamiento regional en lugar del correspondiente al conjunto del Estado.

Siguiendo lo destacado en la literatura especializada, las variables analizadas en este trabajo se han agrupado en torno a cuatro aspectos de gran trascendencia que son: el esfuerzo económico regional, las particularidades de cada RMI, su cobertura y su capacidad protectora (Tabla 1). La escasez de datos adicionales o con más nivel de detalle, así como los propios objetivos de la investigación, no han permitido incorporar otras cuestiones de gran interés, como por ejemplo las relativas a la efectividad de dichas rentas en el fomento de la inserción laboral. La consideración de este asunto, por otro lado, habría requerido de otras metodologías de análisis orientadas a relacionar la percepción de la prestación con las entradas y salidas de los beneficiarios del mercado laboral y de las bolsas de pobreza.

Las principales fuentes estadísticas empleadas han sido el último informe de RMI del Ministerio de Sanidad, Consumo y Bienestar Social (MSCBS), la Contabilidad Regional de 2017, para construir $X_{2}$ y la Encuesta de Condiciones de Vida (ECV) de 2017, para construir $X_{5}, X_{9} y_{10}$. Los datos de partida, todos ellos de carácter oficial, presentan algunas limitaciones que condicionan, lógicamente, el alcance y resultados del trabajo. Así, por ejemplo, las cifras oficiales de perceptores de RMI recogen el número de personas que han recibido la prestación en algún momento del año, contabilizándose un beneficiario cada vez que un usuario participa en el programa. Asimismo, al ser la duración de las prestaciones de cada beneficiario muy diferentes (entre 1 y 12 meses), dado que algunos agotan el tiempo legal y otros no, la composición interna de la población perceptora afecta los datos de gasto por perceptor (AIReF 2019). En definitiva, si bien las variables manejadas permiten caracterizar suficientemente el panorama de las diferencias regionales, no cabe duda de que más y mejor información estadística habría conllevado resultados más precisos. En este sentido, una mayor desagregación de los datos en cuanto al perfil de los perceptores (edad, sexo, nivel de estudios o nacionalidad), sobre los periodos de percepción (en lugar de remitirnos únicamente a la duración máxima), el periodo de permanencia de cada titular (que no tiene por qué coincidir con el periodo 
máximo contemplado), entre otras cuestiones, habrían ciertamente permitido arrojar más luz sobre las tipologías de RMI.

Tabla 1. Relación de los indicadores empleados.

\begin{tabular}{|c|c|}
\hline $\begin{array}{l}\text { Aspecto de las RMI que } \\
\text { caracteriza }\end{array}$ & Indicador \\
\hline \multirow[t]{2}{*}{$\begin{array}{l}\text { Esfuerzo } \\
\text { presupuestario }\end{array}$} & $\begin{array}{l}\mathrm{X}_{1} \text { : Gasto anual en RMI por cada beneficiario de prestación }(€) \text { (benefi- } \\
\text { ciario = titular + dependientes) }\end{array}$ \\
\hline & $\begin{array}{l}\mathrm{X}_{2}: \text { Porcentaje que supone el gasto anual en RMI respecto del PIB re- } \\
\text { gional (\%) }\end{array}$ \\
\hline \multirow[t]{5}{*}{ Características básicas } & $X_{3}$ : Cuantía mínima que recibe un único perceptor $(€)$ \\
\hline & $\begin{array}{l}X_{4} \text { : Cuantía máxima que puede percibir una unidad familiar/de con- } \\
\text { vivencia }(€)\end{array}$ \\
\hline & $X_{5}$ : Duración máxima de la prestación (meses) \\
\hline & $\begin{array}{l}\text { X: Tiempo mínimo de existencia de la unidad de convivencia o fami- } \\
\text { liar (meses) }\end{array}$ \\
\hline & $\mathrm{X}_{7}$ : Tiempo mínimo de residencia en la CCAA (meses) \\
\hline Intensidad protectora & $\begin{array}{l}\mathrm{X}_{8} \text { : Gasto medio por titular respecto a la renta media por hogar de la } \\
\text { comunidad }(\%)\end{array}$ \\
\hline \multirow[t]{2}{*}{ Cobertura } & $\begin{array}{l}\mathrm{X}_{\mathrm{g}} \text { : Número de beneficiarios respecto al número de personas en riesgo } \\
\text { de pobreza }(\%)\end{array}$ \\
\hline & $\begin{array}{l}\mathrm{X}_{10}: \text { Número de beneficiarios respecto al número de personas en riesgo } \\
\text { de pobreza severa }(\%)\end{array}$ \\
\hline
\end{tabular}

Notas: (1) La población en riesgo de pobreza es el porcentaje de personas que viven en hogares cuya renta total equivalente anual está por debajo del $60 \%$ de la renta disponible mediana equivalente regional.

(2) La población en riesgo de pobreza relativa severa es el porcentaje de personas que viven en hogares cuya renta total equivalente anual está por debajo del $25 \%$ de la renta disponible mediana equivalente regional.

\section{Análisis clúster}

El análisis clúster ha sido la técnica empleada para obtener grupos de regiones con una estructura interna homogénea a partir de las 10 variables que caracterizan las RMI. Dado que las unidades de medida de las variables son diferentes, se han convertido a una escala común con la transformación minmax (Everitt et al 2011 proporcionan una introducción detallada sobre el tema). Este artículo utiliza un método jerárquico de forma exploratoria para obtener una aproximación sobre el número razonable de clústeres. En concreto, se utiliza el método de Ward (Ward 1963), que trata de minimizar la variabilidad intra-grupo. Posteriormente, partiendo de los resultados del método de jerárquico, se utiliza un método no jerárquico, el algoritmo de k-medias, con el fin de ajustar o de validar la agrupación realizada (Steinley 2006). En cada etapa del método de k-medias, las regiones se asignan a los grupos en función de lo similar que son las variables de cada región a la semilla o valores de referencia en cada grupo.

Una de las cuestiones más controvertidas es determinar el número de clústeres que recoja la estructura de los datos. El análisis realizado está basado en dos 
herramientas. En primer lugar, se analiza el dendrograma que representa las fusiones que se han hecho en cada etapa y sus correspondientes distancias. También empleamos el índice CH propuesto por Calinski y Harabasz (1974), que compara la variabilidad de las variables clasificadoras entre grupos y la variabilidad de dichas variables dentro de los grupos. ${ }^{6}$

Para concluir esta etapa, se lleva a cabo un análisis de la varianza para comprobar si existen, por término medio, diferencias significativas en los indicadores entre los grupos alcanzados y analizar la importancia relativa de cada variable para discriminar los grupos obtenidos. Asimismo, se obtienen y analizan diferentes estadísticos para encontrar el perfil que definen cada clúster. Los programas informáticos utilizados han sido STATA 15 y SPSS 24.

\section{RESULTADOS}

Antes de presentar los resultados, es importante señalar que, en primer lugar, se ha analizado el grado de correlación entre las variables. ${ }^{7}$ Así, se ha comprobado que las correlaciones entre las variables no son muy elevadas, excepto la que existe entre el porcentaje que supone el gasto anual en RMI respecto del PIB regional $\left(X_{2}\right)$ y el número de beneficiarios respecto al número de personas en riesgo de pobreza $\left(X_{9}\right)$, con un coeficiente de correlación superior a 0.9. Estas dos últimas variables caracterizan dos aspectos diferentes de las RMI, por lo que, a pesar de su alta correlación, se han decidido mantener en el análisis. No obstante, se ha comprobado que los resultados al eliminar una de ellas alteran muy poco la clasificación alcanzada.

El dendrograma, que aparece en el Gráfico 2, muestra las etapas del proceso de agregación de abajo a arriba. Se observa que el principal cambio en los coeficientes de fusión se produce cuando se pasa de 2 clústeres a 1. Otro cambio importante se encuentra en el paso de 4 a 3 clústeres. No obstante, los coeficientes de fusión en las etapas en las que se forman 4, 3 y 2 conglomerados no son excesivamente elevados, pudiendo ser consideradas posibles soluciones. La opción finalmente elegida ha sido la de 4 clústeres, por presentar el índice CH más elevado (7.39 frente a 2.17, de la solución de tres grupos, y 4.36, de la de 2 grupos). 
Gráfico 2. Dendrograma del enlace de Ward con estandarización minmax.

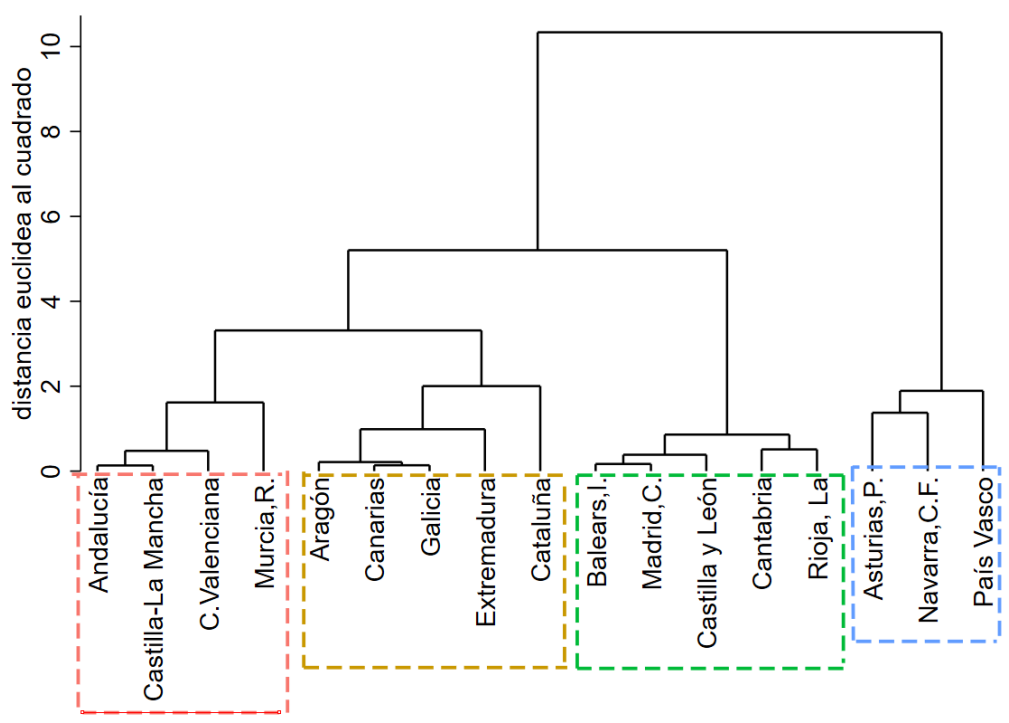

A partir del número de clústeres sugerido por el método de Ward, hemos realizado un análisis de k-medias. Así, al establecer 4 grupos y como semillas iniciales los centroides de los grupos generados por el método de Ward, el resultado de la agrupación de k medias es el mismo que el del método jerárquico, lo que muestra la robustez de la agrupación del método empleado. ${ }^{8}$

Con el fin de encontrar el perfil que caracteriza cada uno de los grupos, la Tabla 2 muestra las regiones pertenecientes a cada grupo, así como los principales estadísticos de las 10 variables. Asimismo, se presenta el estadístico de contraste F del análisis de la varianza. Se confirma la validez de la agrupación realizada dado que se observan diferencias entre los grupos estadísticamente significativas al 5\% en el comportamiento medio de la mayoría de las variables. El número de beneficiarios respecto al número de personas en riesgo de pobreza $\left(X_{9}\right)$ es la variable que contribuye en mayor medida a diferenciar entre los cuatro grupos, le siguen el porcentaje que supone el gasto anual en RMI respecto del PIB regional $\left(X_{2}\right)$ y la duración máxima de la prestación $\left(X_{5}\right)$. La cuantía máxima $\left(X_{4}\right)$, el tiempo mínimo de existencia de la unidad de convivencia o familiar $\left(X_{6}\right)$ y el gasto medio por titular respecto a la renta media por hogar de la comunidad $\left(X_{8}\right)$ son las que presentan menor variabilidad entre los cuatro grupos. 
Tabla 2. Principales estadísticos descriptivos de los indicadores en los clústeres.

\begin{tabular}{|c|c|c|c|c|c|c|c|c|c|c|c|}
\hline Gru & & $X_{1}$ & $X_{2}$ & $X_{3}$ & $X_{4}$ & $X_{5}$ & $X_{6}$ & $X_{7}$ & $X_{8}$ & $X_{9}$ & $X_{10}$ \\
\hline \multirow{4}{*}{$\begin{array}{l}\text { G1: Andalucía; } \\
\text { Castilla-La Mancha; } \\
\text { Com. Valenciana; } \\
\text { Región de Murcia }\end{array}$} & Tedia & 902 & 0.04 & 411 & 703 & 20 & 12 & 30 & 11.00 & 4.57 & 20.07 \\
\hline & Min. & 602 & 0.02 & 389 & 626 & 6 & 0 & 12 & 9.20 & 2.84 & 14.44 \\
\hline & Max. & 1129 & 0.06 & 430 & 807 & 36 & 24 & 60 & 12.40 & 5.76 & 23.10 \\
\hline & $\mathrm{CV}$ & 0.26 & 0.42 & 0.04 & 0.11 & 0.68 & 0.82 & 0.69 & 0.12 & 0.27 & 0.19 \\
\hline \multirow{4}{*}{$\begin{array}{l}\text { G2: Aragón; } \\
\text { Canarias; Cataluña; } \\
\text { Extremadura; Galicia }\end{array}$} & Media & 2310 & 0.14 & 464 & 777 & 24 & 2 & 13 & 21.70 & 7.31 & 40.91 \\
\hline & Min. & 1927 & 0.08 & 403 & 665 & 12 & 0 & 6 & 15.30 & 4.62 & 18.51 \\
\hline & Max. & 2874 & 0.26 & 564 & 1062 & 60 & 12 & 24 & 37.60 & 11.43 & 80.54 \\
\hline & $\mathrm{CV}$ & 0.19 & 0.50 & 0.14 & 0.21 & 0.87 & 2.24 & 0.50 & 0.43 & 0.41 & 0.70 \\
\hline \multirow{4}{*}{$\begin{array}{l}\text { G3: Islas Baleares; } \\
\text { Cantabria; Castilla y } \\
\text { León; Com. de Madrid; } \\
\text { La Rioja }\end{array}$} & Media & 1511 & 0.11 & 424 & 724 & 100 & 7 & 11 & 12.92 & 9.70 & 73.91 \\
\hline & Min. & 780 & 0.04 & 400 & 672 & 100 & 0 & 6 & 5.50 & 5.57 & 23.63 \\
\hline & Max. & 2206 & 0.24 & 430 & 777 & 100 & 12 & 12 & 19.20 & 14.45 & 135.76 \\
\hline & $\mathrm{CV}$ & 0.42 & 0.71 & 0.03 & 0.06 & 0.00 & 0.70 & 0.25 & 0.48 & 0.38 & 0.65 \\
\hline \multirow{5}{*}{$\begin{array}{l}\text { G4: Com. Foral de } \\
\text { Navarra; País Vasco; } \\
\text { Principado de Asturias }\end{array}$} & Media & 2601 & 0.56 & 559 & 8 & 75 & 6 & 28 & 18.63 & 36.84 & 153.00 \\
\hline & Min. & 1743 & 0.50 & 443 & 713 & 24 & 0 & 24 & 18.00 & 32.48 & 127.63 \\
\hline & Max. & 3298 & 0.66 & 635 & 1200 & 100 & 12 & 36 & 19.50 & 40.08 & 200.60 \\
\hline & $\mathrm{CV}$ & 0.30 & 0.15 & 0.18 & 0.26 & 0.59 & 1.00 & 0.25 & 0.04 & 0.11 & 0.27 \\
\hline & $\begin{array}{c}\text { Estadís- } \\
\text { ticoF }\end{array}$ & $7.75^{*}$ & $38.76^{*}$ & $4.88^{*}$ & 1.96 & $14.98^{*}$ & 1.56 & $3.35^{*}$ & 282 & $75.05^{*}$ & $9.46^{*}$ \\
\hline \multirow{4}{*}{ Total } & Media & 1795 & 0.18 & 457 & 772 & 54 & 7 & 19 & 16.06 & 12.58 & 65.49 \\
\hline & Min. & 602 & 0.02 & 389 & 626 & 6 & 0 & 6 & 5.50 & 2.84 & 14.44 \\
\hline & Max. & 3298 & 0.66 & 635 & 1200 & 100 & 24 & 60 & 37.60 & 40.08 & 200.60 \\
\hline & $\mathrm{CV}$ & 0.46 & 1.06 & 0.16 & 0.20 & 0.76 & 1.04 & 0.70 & 0.45 & 0.96 & 0.86 \\
\hline
\end{tabular}

Nota. ${ }^{*}$ Diferencias estadísticamente significativas al 5\%.

El análisis estadístico realizado permite configurar cuatro grupos (o clústeres) de CCAA en base a las características internas de cada grupo y a las diferencias observadas entre ellos: un grupo (G1) con sistemas de RMI que denominaremos "débiles", por presentar los datos más desfavorables en la mayoría de las variables consideradas; dos grupos intermedios con datos que en un caso (G2) se revelan polarizados y en otro resultan más homogéneos (G3); y un grupo (G4) con resultados muy positivos en prácticamente todos los indicadores que permiten hablar de sistemas de RMI "avanzados."

El comportamiento de las regiones en el G1, conformado por Andalucía, Castilla-La Mancha, Comunidad Valenciana y la Región de Murcia, es bastante homogéneo, con coeficientes de variación de las variables consideradas (CV) inferiores a los que se obtienen para el conjunto de CCAA. En este grupo el porcentaje de gasto respecto del PIB regional $\left(X_{2}\right)$ apenas supone el $0.04 \%$, cuando 
en el conjunto del Estado es cuatro veces superior; las personas que reciben las RMI $\left(\mathrm{X}_{9}\right)$ representan el $4.57 \%$ de la población en situación de pobreza, muy alejado del $12.57 \%$ que se alcanza por término medio en las CCAA; y solo 2 de cada 10 personas en situación de pobreza extrema $\left(X_{10}\right)$-personas que no alcanzan el $25 \%$ de la renta mediana equivalente regional- reciben RMI, tres veces menos que la media de todas las regiones (66\%).

En el G2 se incluyen las CCAA de Aragón, Canarias, Cataluña, Extremadura y Galicia, con un comportamiento muy homogéneo de todas ellas a excepción, como explicaremos más adelante, de Cataluña. Este grupo se sitúa en una posición intermedia y su principal peculiaridad, que le diferencia de G3 -también en espacios intermedios-, es que las regiones que lo componen presentan valores internamente polarizados, al sobresalir en algunos datos de forma positiva y en otros de forma negativa. Por el lado positivo, estas cinco CCAA destacan en tres indicadores: la intensidad protectora $\left(X_{8}\right)$, la más elevada de todos los grupos, con una cuantía media por titular que supone el $21.7 \%$ de la renta media por hogar de la comunidad; el tiempo exigido de existencia de la unidad de convivencia $\left(X_{6}\right)$, dado que la mayoría de las regiones de este clúster no impone ningún límite; y el gasto regional por beneficiario $\left(X_{1}\right)$ de 2,310€ anuales, cercano a los valores que registran las regiones del clúster más avanzado (G4). En el lado negativo, este grupo presenta valores próximos al clúster de sistemas débiles de RMI (G1) en la duración máxima de las prestaciones $\left(\mathrm{X}_{5}\right)$ y en las dos variables relacionadas con el grado de cobertura de la población en riesgo de pobreza y en riesgo de pobreza severa $\left(X_{9} y X_{10}\right)$.

El clúster G3 está formado por cinco regiones: Islas Baleares, Cantabria, Castilla y León, Comunidad de Madrid y La Rioja. Este grupo, con un comportamiento interno bastante homogéneo, presenta una situación intermedia en cuanto a la generosidad del sistema de RMI, con datos mejores que G1. Su principal rasgo distintivo es que sus valores no son tan extremos como el clúster G2: la mayoría de las variables se sitúan en torno a los valores medios calculados con todas las regiones. No obstante, debemos apuntar, también, que algunas de las regiones de este grupo destacan en la duración máxima de la prestación $\left(X_{5}\right)$ puesto que no establecen un tiempo máximo de percepción de la RMI. ${ }^{9}$

El clúster G4 lo integran la Comunidad Foral de Navarra, el País Vasco y el Principado de Asturias. Sus registros se sitúan por encima de la media en 9 de las 10 variables consideradas, circunstancia que permite hablar de "sistemas avanzados" de RMI. Sus datos son extremadamente superiores (sobre todo si se compara con el grupo G1) en la mayoría de variables, pero especialmente en dos: la cobertura de la pobreza severa $\left(X_{10}\right)$ se sitúa en este grupo en un $153 \%$, muy por encima de los registros del G3 (80\%), G2 (41\%) y G1 (20\%); y en estas

Hay que señalar que, en la variable duración máxima, se ha asignado el valor 100 meses a las regiones que no presenta límite. 
regiones el 0.5 \% del PIB per cápita se dedica a rentas mínimas de inserción $\left(X_{2}\right)$, muy alejado del $0.26 \%$ en el que se sitúa el resto. Llama la atención, sin embargo, que sus condiciones de acceso son muy exigentes, al requerir más tiempo de residencia en la comunidad que la media y un elevado tiempo de establecimiento de la unidad de convivencia (mayor que en G2).

Como es lógico en un proceso de clasificación de estas características, hay regiones que no se ajustan perfectamente a los grupos asignados; el caso más notable es Cataluña. Esta región evidencia un comportamiento un tanto diferente al de su grupo (G2-intermedio polarizado) porque presenta valores no muy alejados del G3 (intermedio homogéneo) en dos variables con fuerte peso a la hora de discriminar entre grupos: el porcentaje que supone el gasto anual en RMI respecto del PIB regional $\left(\mathrm{X}_{2}\right)$ y la duración máxima de la prestación $\left(X_{5}\right)$. Se encuentra, también, entre las regiones que proporcionan las mayores cuantías a los beneficiarios y los titulares tanto en términos absolutos como relativos. Sin embargo, sus niveles de cobertura y los requisitos bastante restrictivos en cuanto al tiempo de residencia y de la unidad de convivencia provoca que su comportamiento sea más similar al de las comunidades del grupo G2, posición que, por otra parte, resulta congruente con la tesis que defienden algunos trabajos cuando aseveran que el éxito de un programa de RMI no depende tanto de la generosidad de las cuantías cuanto de su concepción y del modelo de gestión implementado.

El diagrama radial del Gráfico 3 elaborado a partir de las medias minmax, permite apreciar gráficamente las fortalezas y debilidades del sistema público de

Gráfico 3. Diagrama radial de los indicadores en los cuatro grupos de regiones.

\begin{tabular}{cccc} 
- Grupo 1 & Grupo 2 & Grupo 3 & Grupo 4 \\
\cline { 2 - 4 } Sistema & Sistema & Sistema & Sistema \\
débil & $\begin{array}{c}\text { intermedio } \\
\text { polarizado }\end{array}$ & intermedio & avanzado \\
& homogéneo &
\end{tabular}

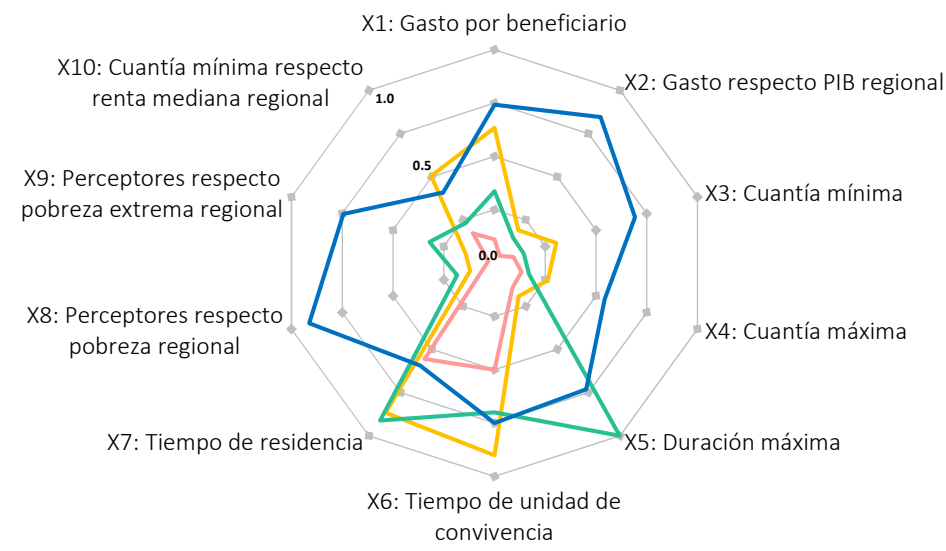


RMI en cada uno de los grupos mencionadas. Se comprueba cómo las regiones encuadradas en G1 serían las que presentan un sistema más frágil o débil, ostentando la conjunción de rasgos más negativos. En G2 y G3, se encontrarían las regiones con posición intermedia, aunque con comportamientos diferentes, como ya se ha mencionado. G4 es el grupo de regiones con el sistema más avanzado o desarrollado, con cifras muy destacadas en los diversos aspectos considerados.

Por último, el Mapa 1 que se presenta a continuación refleja espacialmente la clasificación realizada y apunta a la existencia de una posible relación entre el dinamismo del territorio y las divergencias detectadas. Principalmente llama la atención que las regiones con mejores datos (G4) se encuentran claramente ubicadas en la mitad norte del país, mientras que las que presentan un sistema de RMI menos desarrollado (G1) están en la mitad meridional. Por su parte, las comunidades con sistemas de RMI de tipo intermedio (G2 y G3) forman un bloque en la mitad septentrional de la península, al que se suman Extremadura y las regiones insulares. Para profundizar, no obstante, sobre esta posible relación, se precisaría de otro tipo de análisis que trasciende al alcance de la presente investigación.

Mapa 1. Distribución espacial de los cuatro grupos de regiones.

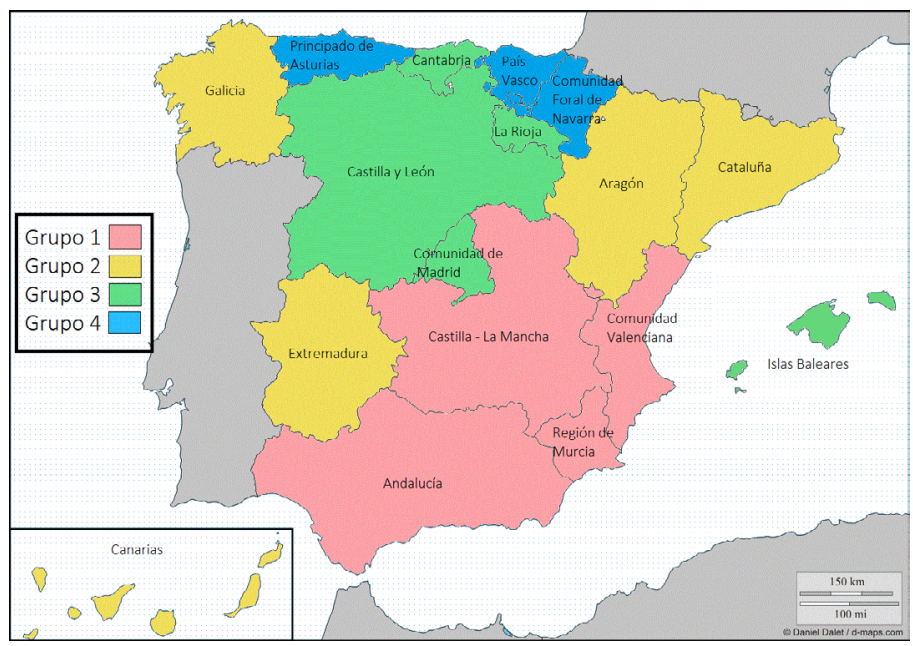

\section{CONCLUSIONES}

El análisis realizado de las diferencias regionales en las características y el alcance de las RMI, una de las herramientas más importantes para combatir la pobreza, ha permitido confirmar lo que muchos otros trabajos anteriores apuntaban: persiste en la actualidad en España un panorama diverso y desigual que 
se explica, en primer lugar, por el diferente esfuerzo realizado por los gobiernos regionales, principales responsables de este recurso, pero también por la ausencia de mecanismos de coordinación y compensación orientados a generar un modelo territorial equilibrado y verdaderamente garante de la igualdad de derechos y oportunidades de todas las personas.

Nuestro trabajo ha prestado atención a algunas de las variables que concretan el esfuerzo presupuestario que hace cada ente regional, a aquellas que caracterizan la naturaleza y extensión que tiene esta prestación en cada territorio, a otras que dimensionan el nivel de adecuación a la población potencialmente destinataria y a las que permiten valorar la intensidad protectora que implica su cuantía de acuerdo a los diferentes umbrales de riesgo de pobreza. Estas variables consideradas de manera aislada permiten observar la enorme heterogeneidad del "mosaico" que constituye la multiplicidad de sistemas de RMI; sin embargo, es el análisis conjunto de todas ellas mediante procedimientos estadísticos (análisis clúster) el que permite dimensionar las disparidades y las confluencias inter e intrarregionales para, desde éstas, configurar las cuatro agrupaciones de CCAA señaladas.

Si bien partíamos de 17 modelos distintos de RMI cuyas diferencias se han ido suavizando a lo largo del tiempo, los resultados del análisis informan de manera consistente de la existencia en la actualidad de cuatro grupos diferenciados de CCAA. Uno de ellos (G4), el integrado por Navarra, País Vasco y Asturias, agrupa a las regiones con mejores condiciones en sus RMI, mayor grado de cobertura de la población necesitada y mayor generosidad en los recursos asignados. En la situación contraria se encuentra el grupo (G1) de CCAA integrado por Andalucía, Castilla-La Mancha, Comunidad Valenciana y Región de Murcia, con un esfuerzo presupuestario e indicadores de cobertura muy por debajo de la media nacional. Entre uno y otro caso, se sitúan otros dos: las CCAA de Aragón, Canarias, Extremadura, Cataluña y Galicia (G2) presentan mejores resultados que el G1, especialmente en el gasto medio por titular, el gasto regional por beneficiario y el tiempo exigido de existencia de la unidad de convivencia; e Islas Baleares, Cantabria, Castilla y León, Comunidad de Madrid y La Rioja (G3), con valores medios en todas las variables consideradas. Este ejercicio clasificatorio y tipológico confirma que no se puede hablar de un sistema español de RMI, pero que tampoco es exacto afirmar que existen 17 programas totalmente diferenciados: la identificación de diferentes grupos pone de manifiesto ciertas pautas y tendencias que comparten algunos programas entre sí, cuestión que ha de ser necesariamente tenida en cuenta de cara a orientar cómo debería procederse a la mejora de su gestión para conseguir una mayor efectividad en el abordaje de la pobreza.

Las RMI, en su concepción y orientación, están llamadas a jugar un importante papel en la lucha contra la pobreza y en el fomento de la inserción laboral de sus perceptores. Sin embargo, su escasa eficacia en la consecución de estos objetivos deriva, por una parte, de las limitaciones y debilidades de las variables que conforman los aspectos considerados en este trabajo -dotaciones 
económicas, criterios de acceso, niveles de cobertura, intensidad protectora- $y$, por otra, de su irregular garantía legal y de la extraordinaria disparidad en la articulación de los procesos de gestión y acompañamiento social que implica su percepción. Afrontar estas debilidades en orden a configurar RMI efectivas en el cumplimiento de sus propósitos, exigirá, entre otras cosas, garantizar su reconocimiento como derecho subjetivo efectivo, comprometer cuantías adecuadas para situar a los beneficiarios por encima del umbral de pobreza y relajar la compatibilidad con otros ingresos salariales hasta un umbral alrededor del salario mínimo, simplificar y suavizar los requisitos para percibirlas, revisar su configuración desde el paradigma de la activación e incrementar los esfuerzos para una mayor individualización. Asimismo, parece inexcusable la adopción de medidas sistémicas que permitan conformar una base estatal común que, respetando la distribución de competencias en la materia, posibilite la adopción de programas más adaptados y adecuados a las exigencias de cada territorio.

Los resultados obtenidos confirman, una vez más, la necesidad de reformar un sistema que, si bien ha cumplido y cumple un papel fundamental en el sostenimiento de las rentas de la población, es claramente mejorable. Los casos de 'éxito relativo', como podría ser el del País Vasco, son un claro referente al que se puede y debe mirar. Pero más allá de reformas concretas en las características de las RMI, lo que verdaderamente se requiere es un debate sobre el modelo global de garantía de renta. Y aquí son varias las opciones a considerar tanto en lo que se refiere a las condiciones de inserción laboral que se van a exigir a los demandantes como en lo que a requisitos de renta se refiere. En ese debate caben también, como no podría ser de otra manera, las voces de los que desde hace tiempo vienen reclamando la incondicionalidad de las prestaciones, es decir, la transición hacia un modelo de renta básica universal.

\section{BIBLIOGRAFÍA}

Aguilar, Manuel, Mario Gaviria y Miguel Laparra. 1994. “Las rentas mínimas de inserción de las Comunidades Autónomas." Documentación Social 96: 201-222.

Aguilar, Manuel y Ana Arriba. 2019. "Crisis económica y transformaciones de la política de garantía de ingresos mínimos para la población activa." Panorama Social 29: 91-106.

AIReF. 2019. Los programas de rentas mínimas en España. Estudio. Madrid: Autoridad Independiente de Responsabilidad Fiscal (AIReF).

Arbelaitz, Olatz, Ibai Gurrutxaga, Javier Muguerza, Jesús M. Pérez e Iñigo Perona. 2013. “An extensive comparative study of cluster validity indices." Pattern Recognition 46: 243256.

Arriba, Ana. 2009. "Rentas Mínimas de Inserción de las Comunidades Autónomas: una visión conjunta de su evolución y alcance." Gestión y Análisis de Políticas Públicas Nueva Época 2: 81-99.

Arriba, Ana. 2014. El papel de la garantía de mínimos frente a la crisis. Madrid: Foessa. VII Informe sobre exclusión y desarrollo social en España 2014, Documento de Trabajo 5.7.

Arriba, Ana y Begoña Pérez. 2007. "La última red de protección social en España: prestaciones asistenciales y su activación." Política y Sociedad 44 (2): 115-133. 
Ayala, Luis. 2016. "El gasto público en programas de lucha contra la pobreza: tendencias, determinantes y necesidades de reforma." Papeles de Economía Española 147: 145-166.

Ayala, Luis, José Mª Arranz, Carlos García y Lucía Martínez. 2016. El sistema de garantía de ingresos en España: tendencias, resultados y necesidades de reforma. Resumen ejecutivo. Madrid: Ministerio de Sanidad, Servicios Sociales e Igualdad.

Barragué, Borja y César Martínez. 2012. El régimen de garantía de ingresos mínimos en España: una propuesta de revisión. Madrid: Fundación Alternativas.

Bergantiños, Noemi, Amaia Bacigalupe y Raquel Font. 2017. “Las rentas mínimas de inserción en época de crisis ¿Existen diferencias en la respuesta de las comunidades autónomas?" Papers. Revista de Sociología 102(3): 399-420.

Calinski, Tadeusz y Joaquim Harabasz. 1974. "A Dendrite Method for Cluster Analysis." Communications in Statistics 3: 1-27.

Casado, David (coord.). 2010. Programa Interdepartamental de la Renda Mínima d'Inserció. Informe final d'avaluació. Barcelona: Institut Català d'Avaluació de Polítiques Públiques.

CES. 2017. Informe sobre políticas públicas para combatir la pobreza en España. Madrid: Consejo Económico y Social.

Constitución Española (1978). Boletín Oficial del Estado, 29 de diciembre de 1978, nº 311, pp. 29313 a 29424.

De la Rica, Sara y Lucía Gorjón. 2017. Assessing the Impact of Minimum Income Scheme in the Basque Country. Bonn: Institute of Labor Economics.

Estepa, Francisco, Mercedes Jiménez y Beltrán Roca. 2019. “La renta mínima de inserción en Andalucía: alcance y eficacia de una prestación nueva." Panorama Social 29: 157-170.

Everitt, Brian S., Sabine Landau, Morven Leese y Daniel Stahl. 2011. Cluster Analysis. Hoboken: John Wiley \& Sons.

Fernández, Guillermo. 2013. “El aumento de la fragilidad de las Rentas Mínimas de Inserción durante la crisis." Documentación Social 166: 169-191.

Galiano, Marta, Reyes de Blas, Fernando Martínez y Nuria Morno-Manzanaro. 2014. "Protección social e ingresos mínimos garantizados." Revista Cauces 28: 55-69.

García, Nicolás, Gabriela Jorquera y María J. Muñoz. 2013. Informe de la renta mínima en la Comunidad de Madrid. Madrid: EAPN-Madrid.

García, Gustavo, Luís Barriga, José M. Ramírez, Arantza Zubiría, Laura Velasco, Alba Álvarez y Ana Aranda. 2019. Índice DEC 2018 de desarrollo de los servicios sociales. Madrid: Asociación Estatal de Directores y Gerentes en Servicios Sociales.

Herranz, Rubén. 2017. "Servicios sociales y rentas mínimas de inserción de las Comunidades Autónomas." En El sistema de protección social en España, editado por Enrique Martín-Serrano. Madrid: CCOO, Cap. 5: 83-92.

Laparra, Miguel y Luis Ayala. 2009. El sistema de garantía de ingresos mínimos en España y la respuesta urgente que requiere la crisis social. Madrid: Cáritas y Fundación Foessa.

Laparra, Miguel. 2013. "La garantía de unos ingresos mínimos para todos: una reforma necesaria para mantener la cohesión social y preservar el capital humano." Cuadernos del Círculo Cívico de Opinión 10: 11-40.

Laparra, Miguel. 2020, 15 de mayo. "Complementariedad o desmantelamiento de las rentas mínimas autonómicas." Agenda pública. Recuperado el 22 de mayo de 2020 de http:/ / agendapublica.elpais.com/complementariedad-o-desmantelamiento-de-las-rentas-minimas-autonomicas /

Lasheras, Rubén, Edurne Jabat, Madalena d'Oliveira-Martins e Ignacio Sánchez. 2017. “Efectos desintegradores de las políticas de integración: la Renta de Inclusión Social en Navarra." Cuadernos de Trabajo Social 30(2): 315-327.

Malgesini, Graciela. 2014. Informe sobre los Sistemas de Rentas Mínimas en España. Comisión Europea.

Martínez, María. 2005. “Las rentas mínimas autonómicas desde una perspectiva comparada." Cuadernos de Relaciones Laborales 23(2): 151-189.

Mato, Francisco J. 2019. "Crisis, recuperación y flujos de entradas y salidas en programas de rentas mínimas: el caso de Asturias." Panorama Social 29: 137-156. 
Milligan, Glenn W. y Martha C. Cooper. 1985. “An Examination of Procedures for Determining the Number of Clusters in a Data Set." Psychometrika 50: 59-179.

Ministerio Sanidad, Consumo y Bienestar Social. 2019. El sistema público de Servicios Sociales. Informe de rentas mínimas de inserción. Madrid: MSCBS.

Montes, Andoni. 2020, 25 de mayo. "Redibujar las políticas de protección social en un Estado descentralizado." Agenda Pública. Recuperado el 25 de mayo de 2020 de http:/ /agendapublica.elpais.com/redibujar-las-politicas-de-proteccion-social-en-un-estado-descentralizado/

Moreno, Ana. 2018. "Requisitos comunes para el acceso a las prestaciones autonómicas de garantía de ingresos." Temas Laborales 143: 87-123.

Mooi, Erik y Marko Sarstedt. 2011. "Chapter 9: Cluster Analysis." En A Concise Guide to Market Research, editado por Erik Mooi y Marko Sarstedt. Berlin: Springer-Verlag, 273-324.

Moreno, Gorka y Xabier Aierdi. 2019. “La Renta de Garantía de Ingresos vasca: entre la necesidad y la eficacia." Panorama Social 29: 171-182.

Noguera, José A. 2018, 29 de abril. "La nueva generación de rentas mínimas: ¿hacia una renta garantizada?" Recuperado el 26 de julio de 2019 de http:/ /agendapublica.elpais. com/la-nueva-generacion-de-rentas-minimas-hacia-una-renta-garantizada/

Noguera, José A. 2019. “Las rentas mínimas autonómicas en España: balance y retos de futuro." Observatorio Social La Caixa. Las rentas mínimas autonómicas en España: balance y retos de futuro. Colección Informes, septiembre.

Riba, Clara, Xavier Ballart, Jaume Blasco. 2011. "Minimum Income and Labour Market Integration Processes: Individual and Institutional Determinants." Revista Española de Investigaciones Sociológicas 133: 43-58.

Rodríguez, Gregorio. 2009. Valoración de los programas de rentas mínimas en España. Bruselas: European Commission. DG Employment, Social Affairs and Equal Opportunities.

Sanzo, Luis. 2013. "La política de garantía de ingresos en Euskadi." Zerbitzuan 53: 9-28.

Sanzo, Luis. 2018a. "La política de garantía de ingresos en España." Zerbitzuan 65: 41-51.

Sanzo, Luis. 2018b. "Empleo y garantía de ingresos en España." En Bienestar social y políticas públicas. Retos para pactar el futuro, editado por Germán Jaraíz. Madrid: Catarata, 203220.

Sanzo, Luis. 2020, 15 de junio. “Ingreso Mínimo Vital y rentas mínimas autonómicas. ¿Qué harán las Comunidades Autónomas?" Recuperado el 15 de junio de https://hungrygrass.files.wordpress.com/2020/06/ingreso-minimo-vital-y-vias-de-ajuste-para-las-rentas-minimas-autonomicas.pdf

Steinley, Douglas. 2006. "K-means Clustering: a Half-century Synthesis." British Journal of Mathematical and Statistical Psychology 59:1-34.

Valdueza, María D. 2019. “La garantía de ingresos mínimos de las comunidades autónomas: prestación económica e inserción sociolaboral." Panorama Social 29: 51-69.

Ward, Joe H. 1963. "Hierarchical Grouping to Optimize an Objective Function." Journal of the American Statistical Association 58: 236-244.

Zalakaín, Joseba. 2014. "El papel de los sistemas de garantía de ingresos en el abordaje de la pobreza en el empleo: la experiencia del País Vasco." Lan Harremanak 31: 36-62.

Recibido: 26 de diciembre de 2019

Aceptado: 4 de septiembre de 2020 
Carmen Rodríguez Sumaza. Licenciada en Ciencias Económicas y Empresariales, Doctora en Sociología y Profesora Titular de Sociología en la Universidad de Valladolid (España). Sus principales áreas de investigación son: bienestar y política social, población, género y familia. Actualmente participa en varios proyectos de investigación sobre evaluación de programas sociales y sobre reorganización de los cuidados a las personas mayores. Email: sumaza@eco.uva.es.

María de las Mercedes Prieto Alaiz. Licenciada en Ciencias Económicas y Empresariales (1988), Master of Science in Statistics (1995) por la London School of Economics (LSE) y Doctora en Economía por la Universidad de Valladolid (1998). Su investigación, desde el Departamento de Economía Aplicada (Estadística y Econometría), se centra en la desigualdad, pobreza, distribución de la renta y precariedad laboral, con 12 artículos publicados en 8 revistas indexadas en JCR y 2 artículos en Scopus. Email: prietoal@eco.uva.es.

Juan María Prieto Lobato. Diplomado en Trabajo Social, Doctor en Sociología (mención internacional; Doctor Europeus) y Profesor Titular de Universidad en el Área de Trabajo Social y Servicios Sociales de la Universidad de Valladolid. En la actualidad participa en varios equipos de investigación sobre evaluación de programas sociales (de atención a personas mayores en su domicilio y de cuidados al final de la vida), políticas de inclusión en España y Tercer Sector de Acción Social. E-mail: juanmaria.prieto@uva.es.

Jesús García-Araque. Doctor en Geografía y Doctorando en Economía, docente e investigador en el Departamento de Geografía de la Universidad de Valladolid, premio extraordinario al mejor expediente en el Grado de Geografía y en el Máster en Desarrollo Económico Regional y Local y Gestión del Territorio. Desde una perspectiva multidisciplinar, sus áreas de investigación son: políticas sociales, exclusión social, vulnerabilidad urbana y cartografía participativa. Email: jesus. garcia@uva.es 


\section{ANEXO.}

Valores de las variables empleados en el análisis clúster

\begin{tabular}{lcccrrrrrrr}
\hline CCAA & $\mathrm{X}_{1}$ & $\mathrm{X}_{2}$ & $\mathrm{X}_{3}$ & $\mathrm{X}_{4}$ & $\mathrm{X}_{5}$ & $\mathrm{X}_{6}$ & $\mathrm{X}_{7}$ & $\mathrm{X}_{8}$ & $\mathrm{X}_{9}$ & $\mathrm{X}_{10}$ \\
\hline Andalucía & 841 & 0,06 & 406 & 655 & 6 & 12 & 12 & 12,4 & 5,8 & 23,1 \\
\hline Aragón & 1.959 & 0,14 & 472 & 707 & 12 & 0 & 12 & 16,2 & 11,4 & 80,5 \\
\hline Asturias, P. & 1.743 & 0,52 & 443 & 713 & 100 & 6 & 24 & 19,5 & 40,1 & 127,6 \\
\hline Balears, I. & 1.012 & 0,04 & 430 & 777 & 100 & 6 & 6 & 5,5 & 5,6 & 46,2 \\
\hline Canarias & 2.091 & 0,12 & 477 & 665 & 24 & 0 & 12 & 17,2 & 5,2 & 18,5 \\
\hline Cantabria & 2.206 & 0,24 & 430 & 762 & 100 & 12 & 12 & 18,1 & 14,5 & 135,8 \\
\hline Castilla y León & 2.121 & 0,14 & 430 & 699 & 100 & 0 & 12 & 19,2 & 7,8 & 51,1 \\
\hline Castilla-La Mancha & 602 & 0,02 & 420 & 723 & 24 & 12 & 24 & 9,2 & 2,8 & 14,4 \\
\hline Cataluña & 2.700 & 0,08 & 564 & 1.062 & 60 & 12 & 24 & 22,2 & 4,6 & 23,3 \\
\hline C. Valenciana & 1.129 & 0,05 & 389 & 626 & 36 & 24 & 24 & 11,3 & 4,6 & 21,8 \\
\hline Extremadura & 2.874 & 0,26 & 403 & 726 & 12 & 0 & 12 & 37,6 & 9,6 & 61,9 \\
\hline Galicia & 1.927 & 0,10 & 403 & 726 & 12 & 0 & 6 & 15,3 & 5,7 & 20,3 \\
\hline Madrid, C. & 1.436 & 0,08 & 400 & 708 & 100 & 6 & 12 & 14,6 & 7,9 & 23,6 \\
\hline Murcia, R. & 1.036 & 0,05 & 430 & 807 & 12 & 0 & 60 & 11,1 & 5,1 & 20,9 \\
\hline Navarra, C. F. & 2.762 & 0,50 & 600 & 1.200 & 100 & 0 & 24 & 18,4 & 32,5 & 130,8 \\
\hline País Vasco & 3.298 & 0,66 & 673 & 996 & 24 & 12 & 36 & 18,0 & 37,9 & 200,6 \\
\hline Rioja, La & 780 & 0,06 & 430 & 672 & 24 & 12 & 12 & 7,2 & 12,7 & 112,9 \\
\hline
\end{tabular}

